\title{
7. Harmonic Measures and Capacity of Sets of the Ideal Boundary. II
}

\author{
By Zenjiro KURAMOCHI \\ Mathematical Institute, Osaka University \\ (Comm. by K. KunUGI, M.J.A., Jan. 12, 1955)
}

Let $R$ be a positive boundary Riemann surface and let $D^{1)}$ be a non compact domain determining a subset $B_{D}$ of the ideal boundary. Put $D_{n}=\left(R-R_{n}\right) \cap D$. Let $U_{n, n+i}(z)$ be a harmonic function in $R_{n+i}-$ $R_{0}-D_{n}$ such that $U_{n, n+i}(z)=0$, on $\partial R_{0}, U_{n, n+i}(z)=1$ on $\partial D_{n}$ and $\frac{\partial U_{n, n+i}}{\partial n}=0$ on $\partial R_{n+i}-D_{n}$. Then $\lim _{n=\infty} \lim _{i=\infty} U_{n, n+i}(z)=\lim _{n=\infty} U_{n}(z)=U(z)$, where $U(z)$ is the equilibrium potential of $B_{D}$. We have proved that

$$
\int_{\partial R_{n}} \frac{\partial U_{n}}{\partial n} d s=\int_{\partial G_{\varepsilon}} \frac{\partial U_{n}}{\partial n} d s
$$

for every $G_{\varepsilon}$ except for at most one $\varepsilon$, where $G_{\varepsilon}$ is the domain in which $U_{n}(z)>1-\varepsilon$. Let $U_{n, n+i}^{\prime}(z)$ be a harmonic function in $R_{n+i}-G_{\varepsilon}-R_{0}$ such that $U_{n, n+i}^{\prime}(z)=0$ on $\partial R_{0}, \quad U_{n, n+i}^{\prime}(z)=1-\varepsilon$ on $\partial G_{\varepsilon} \cap R_{n+i}$ and $\frac{\partial U^{\prime}{ }_{n, n+i}}{\partial n}=0$ on $\partial R_{n+i}-G_{\varepsilon}$. Then $\lim _{i=\infty} U^{\prime}{ }_{n, n+i}(z)=U_{n}(z)$.

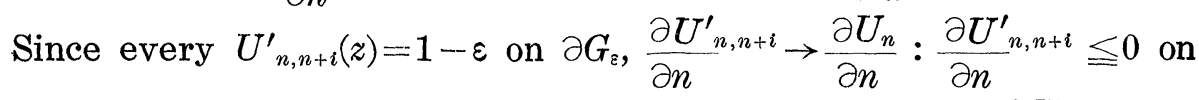
every point of $\partial G_{\varepsilon} \cap R_{n+i}$. Hence by (1) and $\lim _{i=\infty} \int_{\partial R_{0}} \frac{\partial U_{n}, n+i}{\partial n} d s=$ $\int_{\partial R_{0}} \frac{\partial U_{n}}{\partial n} d s$, we easily that

$$
\lim _{i=\infty} \int_{\partial G_{\varepsilon}} \varphi_{i} \frac{\partial U^{\prime}{ }_{n, n+i}}{\partial n} d s=\int_{\partial G_{\varepsilon}} \varphi \frac{\partial U_{n}}{\partial n} d s
$$

on $\partial G_{\varepsilon}$ for every bounded sequence of continuous functions $\varphi_{i} \rightarrow \varphi$ : $\left|\varphi_{i}\right| \leqq \underline{M}<\infty$.

We denote by $G_{n}$ the domain in which $U_{n}(z)>1-\varepsilon_{n}$, where $\varepsilon_{1}>\varepsilon_{2}>\cdots ; \lim \varepsilon_{n}=0$ and every $\varepsilon_{n}$ satisfies the condition (1).

Let $U^{\prime \prime}{ }_{n, n+i}^{n}(z)$ be a harmonic function in $R_{n+i}-R_{0}-G_{n}$ such that $U_{n, n+i}^{\prime \prime}(z)=U(z)$ on $\partial G_{\varepsilon}+\partial R_{0}$ and $\frac{\partial U_{n, n+i}^{\prime \prime}}{\partial n}=0$ on $\partial R_{n+i}-G_{n}$. Since $U_{n}(z)$ is the function such that $U_{n}(z)=1-\varepsilon_{n}$ and $U_{u}(z)$ has the minimum Dirichlet integral over $R-R_{0}-G_{n}$, and since $\lim _{n=\infty} U_{n}(z)=U(z)$ on $\partial G_{n}$, then by (2) we can prove as in the previous paper ${ }^{2)}$

$$
\lim _{n=\infty} \lim _{i=\infty} U_{n, n+i}^{\prime \prime}(z)=U(z) .
$$

I) See, the definition of non compact domain. "Harmonic measures and capacity.

2) See (1). 
Hence we have the following

\section{Lemma.}

$$
U(z)=U_{e x}(z)
$$

where the extremisation is with respect to the sequence $\left\{G_{n}\right\}$.

Now we apply Green's formula to $U_{n, n+i}^{\prime}(z)$ and $U_{n, n+i}^{\prime \prime}(z)$. Then

$$
\begin{aligned}
& \int_{\partial R_{0}+\left(\partial G_{n} \cap R_{n+i}\right)+\left(\partial R_{n+i}-G_{n}\right)} U_{n, n+i}^{\prime}(z) \frac{\partial U_{n, n+i}^{\prime \prime}}{\partial R_{0+}\left(\partial G_{n} \cap R_{n+i}\right)+\left(\partial R_{n+i}-G_{n}\right)} U_{n}^{\prime \prime} \\
& \left(1-\varepsilon_{n}\right) \int_{\partial R_{0}} \frac{\partial U_{n, n+i}^{\prime \prime}}{\partial n} d s=\int_{\partial G_{n} \cap R_{n+i}} U_{n, n+i}^{\prime \prime}(z) \frac{\partial U^{\prime}}{\partial n}{ }_{n, n+i}^{\prime} d s .
\end{aligned}
$$

Let $i \rightarrow \infty$. Then by (1) and (2) we have

$$
\left(1-\varepsilon_{n}\right) \int_{\partial R_{0}} \frac{\partial U}{\partial n} d s=\int_{\partial G_{n}} U(z) \frac{\partial U_{n}}{\partial n} d s .
$$

On the other hand, since $\lim _{n=\infty} \int_{\partial R_{0}} \frac{\partial U_{n}}{\partial n} d s=\operatorname{Cap}\left(B_{D}\right)=\int_{\partial R_{0}} \frac{\partial U}{\partial n} d s$ and $\left(1-\varepsilon_{n}\right) \int_{\partial R_{0}} \frac{\partial U_{n}}{\partial n} d s=\int_{\partial G_{n}} U_{n}(z) \frac{\partial U_{n}}{\partial n} d s$, we have by $(3)$

$$
\lim _{n=\infty} \int_{\partial G_{n}}\left(U_{n}(z)-U(z)\right) \frac{\partial U_{n}}{\partial n} d s=\lim _{n=\infty}\left(1-\varepsilon_{n}\right) \int_{\partial G_{0}}\left(\frac{\partial U_{n}}{\partial n}-\frac{\partial U}{\partial n}\right) d s=0 .
$$

Since $\lim _{i=\infty} D\left(U_{R_{n+i}-R_{0}-G_{n}}^{\prime}{ }_{n, n+i}(z)\right)=\underset{R-R_{0}-G_{n}}{D}\left(U_{n}(z)\right)$ and $\lim _{i=\infty} D\left(U_{R_{n+i}-R_{0}-\iota_{n}}^{\prime \prime}{ }_{n, i+i}(z)\right)=\underset{R-R_{0}-G_{n}}{D}(U(z))$, we have

$$
\begin{gathered}
\left.\underset{R-R_{0}-G_{n}}{D\left(U_{n}(z)-U(z),\right.} U_{n}(z)\right)=\lim _{i=\infty} D\left(U_{R_{n+i}-R_{0}-G_{n}}^{\prime}{ }_{n,+i}(z)-U_{n, n+i}^{\prime \prime}(z), U_{n, n+i}(z)\right) \\
=\int_{\partial G_{n}}\left(U_{n}(z)-U(z)\right) \frac{\partial U_{n}}{\partial n} d s .
\end{gathered}
$$

Hence by (4) $\quad \lim _{n=\infty} \underset{R-B_{0}-G_{n}}{D}\left(U_{n}(z)-U(z), U_{n}(z)\right)=0$. Thus

$$
\begin{aligned}
& \underset{R-R_{0}-G_{n}}{D}\left(U_{n}(z)-U(z)\right)=\underset{R-R_{0}-G_{n}}{D}(U(z))-D\left(U_{n}(z)\right) \text {, whence } \\
& \lim _{i=\infty} \underset{R-R_{0}-G_{n}}{D}(U(z)) \geqq \lim _{\substack{n=\infty \\
R-R_{0}-G_{n}}} D\left(U_{n}(z)\right) .
\end{aligned}
$$

Since $\underset{G_{n}-D_{n}}{D}\left(U_{n}(z)\right)=\underset{R-R_{0}-D_{n}}{D}\left(U_{n}(z)\right)-\underset{R-R_{0}-G_{n}}{D}\left(U_{n}(z)\right)=\varepsilon_{n} \int_{\partial R_{0}} \frac{\partial U_{n}}{\partial n} d s$,

$$
\lim _{n=\infty} \underset{G_{n}-D_{n}}{D}\left(U_{n}(z)\right)=0
$$

From the Fatou's Lemma, we have

$$
\underset{R-R_{0}}{D}(U(z))=D_{R-R_{0}}\left(\lim _{n=\infty} U_{n}(z)\right) \leqq \lim _{n=\infty}\left(D\left(U_{R-R_{0}-D_{n}}(z)\right)=\operatorname{Cap}\left(B_{D}\right)\right.
$$

Therefore by (5) and (6), we have $\lim _{n=\infty} \underset{G_{n}-I_{n}}{D}(U(z))=0$. Hence

$\lim _{n=\infty} \underset{R-G_{n}-R_{0}}{D}\left(U_{n}(z)\right)=\lim _{n=\infty} \underset{R-G_{n}-R_{0}}{D}(U(z)), \quad \lim _{n=\infty} \underset{G_{n}-D_{n}}{D}\left(U_{n}(z)\right)=\lim _{n=\infty} \underset{G_{n}-D_{n}}{D}(U(z))=0$ and

Therefore

$$
\lim _{n=\infty} \underset{G_{n}-D_{n}}{D}\left(U_{n}(z)-U(z), \quad U_{n}(z)\right)=0
$$




$$
\lim _{n=\infty} \underset{R-R_{0}}{D}\left(U_{n}(z)-U(z)\right)=\lim _{n=\infty}\left(D \left(U_{R-G_{n}}\left(U_{n}(z)-U(z)\right)+\underset{G_{n}-D_{n}}{D}\left(U_{n}(z)-U(z)\right)=0 .\right.\right.
$$

It follows that $U_{n}(z)$ converges to $U(z)$ in norm. Then we have the following

Proposition. $\operatorname{Cap}\left(B_{D}\right)=\int_{\partial R_{0}} \frac{\partial U}{\partial n} d s=\underset{R-R_{0}}{D}(U(z))$.

The extremisation is defined with respect to the sequence $\left\{G_{n}\right\}$, we can also the above operation with respect to $\left\{D_{n}\right\}$.

Every $U_{m}(z)(m=n, n+1, \ldots)\left(U(z)=\lim U_{n}(z)\right)$ is the harmonic function which has the minimum Dirichlet integral over $R-R_{0}-D_{n}$ among all functions which have their boundary value $U_{m}(z)$ on $\partial D_{n}$. Let $h(z)$ be a harmonic function in $R-R_{0}-D_{n}$ such that $h(z)=0$ on $\partial D_{n}+\partial R_{0}$ and $\underset{R-D_{n}}{D}(h(z)) \leqq M<\infty$. Then

$$
D\left(U_{m}(z) \pm \varepsilon h(z)\right) \leqq D\left(U_{m}(z)\right) \pm 2 \varepsilon D\left(U_{m}(z), h(z)\right)+\varepsilon^{2} D(h(z)),
$$
whence

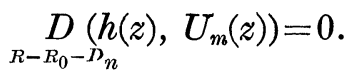

Let $\widetilde{U}_{n}(z)$ be a harmonic function in $R-R_{0}-D_{n}$ such that $\widetilde{U}_{n}(z)=U(z)$ on $\partial D_{n}+\partial R_{0}$ and $\hat{U}_{n}(z)$ has the minimum Dirichlet integral over $R-R_{0}-D_{n}$.

Then

$$
\underset{R-D_{n}-R_{0}}{D}\left(\tilde{U}_{n}(z)\right) \leqq \underset{\bar{R}-R_{0}}{D}(U(z)) \text { and } \underset{R-R_{0}-D_{n}}{D}\left(\tilde{U}_{n}(z), h(z)\right)=0 .
$$

Since $\lim _{n=\infty} U_{n}(z)=U(z)$ on $\partial D_{n}$ and $\lim _{n=\infty} D\left(U_{n-R_{0}-D_{n}}(z)-U(z)\right)=0$,

we can assume $h(z)=\tilde{U}_{n}(z)-U(z)$. Then we have

$$
\lim _{m=\infty}\left[D\left(U(z)-U_{m}(z), h(z)\right)\right]^{2} \leqq \lim _{m=\infty}\left[\underset{R-R_{0}-D_{n}-D_{n}}{D}(h(z)) \underset{R-R_{0}-D}{D}\left(U(z)-U_{m}(z)\right)\right]=0 .
$$

Hence $\left.\underset{R-R_{0}-D_{n}}{D}(z), h(z)\right)=0$, therefore

$$
0=\underset{R-R_{0}-D_{n}}{D}\left(\boldsymbol{U}(z)-\widetilde{U}_{n}(z), h(z)\right)=\underset{R-R_{0}-D_{n}}{D}\left(U(z)-\widetilde{U}_{n}(z)\right) \text {, whence } U(z)=\widetilde{U}_{n}(z) .
$$

Thus we have the next

\section{Theorem 4.}

$$
U(z)=U_{e x}(z),
$$

where the extremisation is defined with respect to the sequence $\left\{D_{n}\right\}$.

Corollary 1. If $U(z) \neq 0, \varlimsup_{z \in D} U(z)=1$.

Proof. Let $\hat{U}_{n, n+i}(z)$ be a harmonic function in $R_{n+i}-R_{0}-D_{n}$ such that $\hat{U}_{n, n+i}(z)=U(z)$ on $\partial D_{n} \cap R_{n+i}, \hat{U}_{n, n+i}(z)=0$ on $\partial R_{0}$ and $\frac{\partial \hat{U}_{n, n+i}}{\partial n}=0$ on $\partial R_{n+i}-D_{n}$. Then $\tilde{U}_{n}(z)=\lim _{i=\infty} \hat{U}_{n, n+i}(z)$. Assume $U(z) \leqq K<1$ on $D$. Then $\hat{U}_{n, n+i}(z) \leqq K U_{n, n+i}(z)$. Hence

$$
U(z)=\lim _{n=\infty} \lim _{i=\infty} \hat{U}_{n, n+i}(z) \leqq K \lim _{n=\infty} \lim _{i=\infty} U_{n, n+i}(z)=K U(z) .
$$


This is absurd. Hence $\varlimsup_{z \in D} U(z)=1$.

Corollary 2. If $U(z) \stackrel{z \in D}{\approx} \approx$, then $\varlimsup_{\lim } U(z)=1$ in $B_{D}$ except possibly for a subset of $B_{D}$ of outer capacity zero.

We denote by $J_{\lambda}(\lambda<1)$ the domain where $U(z)<\lambda$. Put $D \cap J_{\lambda}=H_{\lambda}$. Then $H_{\lambda}$ is a non compact domain determining a subset $I_{\lambda}$ of $B_{D}$. Let $U_{I}(z)$ be the equilibrium potential of $I_{\lambda}$. Then it is clear that $U_{I}(z) \leqq U(z)$. Hence $\varlimsup_{z \in H_{\lambda}} U_{I}(z) \leqq \lambda$. Therefore by the above corollary $U_{I}(z) \equiv 0$.

\section{On the Behaviour of the Green's Function in the Neighbourhood} of the Ideal Boundary

Let $G\left(z, z_{0}\right)$ be the Green's function of $R$ and let $M$ be sufficiently large number. Then $G_{M}=\varepsilon_{z i}\{G(z, z)>M\}$ is compact. We can suppose $R_{0}=G_{M}$. If we consider $R-R_{0}$ as a non compact domain $D$ defining all ideal boundary of $R$. Then it is clear that

$$
1-\frac{G\left(z, z_{0}\right)}{M}=U(z)=\omega^{\prime}(z),
$$

where $U(z)$ and $\omega^{\prime}(z)$ is the equilibrium potential and harmonic measure. Then by the corollary $U(z)=1$ except possibly a subset of ideal boundary of capacity zero. Let $D_{\lambda}=\varepsilon_{z}\{U(z)>\lambda\}$ be a non compact domain determining $B_{D}$. Let $U_{\lambda}(z), \omega_{\lambda}^{\prime}(z)$ and $\omega_{\lambda}(z)$ be equilibrium potential of $B_{D}$ and harmonic measures. Then $0=U_{\lambda}(z)=\omega_{\lambda}^{\prime}(z)$ and $\omega_{\lambda}(z)=0$ is equivalent to $\omega(z)=0$. Thus we have the next

Theorem 5. Cap $\left(B_{n}\right)=0=\omega_{\lambda}(z)$.

We can construct an open Riemann surface $\hat{D}_{\lambda}$ by the process of symmetrization with respect to $\partial D_{\lambda}$. Then we have the following

Corollary. $D_{\lambda}+\hat{D}_{\lambda}$ is a null-boundary Riemann surface.

Proof. Let $\omega_{n}(z)$ be the harmonic measure of $\left(\partial R_{n} \cap D_{\lambda}\right)+\left(\partial R_{n} \cap D_{\lambda} \hat{)}\right.$ with respect to $\left(\left(D_{\lambda} \cap R_{n}\right)-R_{0}\right)+\left(\left(D_{\lambda} \cap R_{n}\right)-R_{0}\right)$. Then $\omega_{n}(z)=0$ on $\partial R_{0}+\partial \hat{R_{0}}, \omega_{n}(z)=1$ on $\left(\partial R_{n} \cap D_{\lambda}\right)$ and $\frac{\partial \omega_{n}}{\partial n}=0$ on $\partial D_{\lambda}$. On the other hand let $U_{n, n+i}(z)$ be a function in $\left(D_{\lambda} \cap R_{n}\right)-R_{0}$ such that $U_{n, n+\varepsilon}(z)=0$ on $\partial R_{0}, U_{n, n+i}(z)=1$ on $\partial D_{\lambda} \cap\left(R-R_{n}\right)$ and $\frac{\partial U_{n, n+i}}{\partial n}=0$ on $\partial R_{n+i}-D_{\lambda}$.

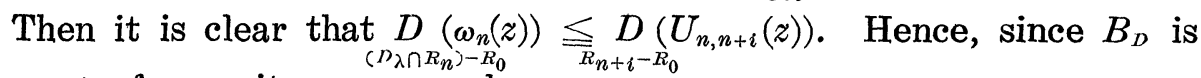
a set of capacity zero, we have

$$
\underset{D_{\lambda} \cap R-R_{0}}{D}\left(\lim _{n}(z)\right) \leqq D\left(\lim _{n} \lim _{i=\infty} U_{n, n+i}(z)\right)=0 .
$$

Thus $D_{\lambda}+\hat{D_{\lambda}}$ is a null-boundary Riemann surface.

Corollary. Let $G\left(z, z_{0}\right)$ be the Green's function of $R$ and let $h(z)$ 
be its conjugate. Put $W(z)=e^{-G\left(z, z_{0}\right)-i h(z)}=r e^{i \theta}$. We cut $R$ along the trajectories $(h(z)=$ const) so that $W(z)$ may be single valued. Then $R$ is mapped onto the domain $|W(z)|<1$ with enumerably infinite number of radial slits. Then $z=z^{-1}(W)$ can be continued analytically along radii re $^{i 0}$ from $W=0$ to $|W|=1$ except possibly a set of $\theta$ of angular measure zero.

In fact, if it were not so, there exists a set $I_{\lambda}$ of the ideal boundary such that $I_{\lambda}$ is defined by a non compact domain $D_{\lambda}=\underset{z}{\varepsilon}$ $\{G(z, z)>\lambda\}$ and the length of the image of $C$ enclosing $I_{\lambda}$ is larger than $l(>0)$. Since Cap $\left(I_{\lambda}\right)=0$, there exists a harmonic function $U_{n}(z)$ in $R-\left(R_{n} \cap D_{\lambda}\right)-R_{0}$ such that $\int_{C_{\mu}} \frac{\partial U_{n}}{\partial n} d s=2 \pi$ and $U_{n}(z)=M_{n}$ $\left(\lim _{n=\infty} M_{n}=\infty\right)$ on $\partial R_{n} \cap D$, where $C \mu=\varepsilon_{z}\left\{U_{n}(z)=\mu\right\}$. Thus by usual method we can deduce a contradiction. Analogously we have

Corollary. If the analytic function $f(z)$ satisfies $D_{R}(f(z))<\infty$. Then the length of the image of trajectories mapped by $f(z)$ is finite for almost $\theta$.

\section{Applications to the Subregion on an Abstract Riemann Surface}

Let $D$ be a non compact domain in $R$. If any bounded (Dirichlet Bounded) harmonic function vanishing on $\partial D$ or having vanishing normal derivative on $\partial D$ must reduce to a constant, we denote by $S_{0 B}, S_{0 D}, S_{0 N B}$ and $S_{0 N D}$ such class of $D$ respectively. In the previous paper, ${ }^{3)}$ we have proved that, if $D$ can be mapped onto a bounded domain then, $S_{0 N B} \subset S_{0 B}$.

Theorem 6. If the genus of $D$ is finite, then $D \in S_{0 N B}=S_{0 N D}$ is equivalent to that $D+\hat{D}$ is a null-boundary Riemann surface.

Proof. If $D+\hat{D}$ is a null-boundary Riemann surface, it is clear that $D \in S_{0 N B}\left(S_{0 N D}\right)$. By assumption, we can suppose $D-R_{n_{0}}$ is a planer surface. Assume $D+\widehat{D}$ is a positive boundary Riemann surface. Then the harmonic measure $\omega(z)$ of the ideal boundary of $\left(D-R_{n_{0}}\right)+$ $\left(D-R_{n_{0}} \hat{)}\right.$ is non-constant. Normalize $\omega(z)$ so that $\int_{\partial R_{0}} \frac{\partial \omega^{\prime}(z)}{\partial n}=2 \pi$ and let $h(z)$ be its conjugate. Then $e^{\omega^{\prime}(z)+i h(z)}=W(z)$ maps $D-R_{n_{0}}$ onto the domain $1<|W|<K$ with enumerably infinite number of radial slits which are the images of $\partial D$ such that $\partial R_{n_{0}}$ is mapped onto $|W|=1$ and $\left(D-R_{n_{0}} \hat{)}\right.$ is symmetric to $\left(D-R_{n_{0}}\right)$ with respect to these slits.

Let $D_{\lambda}(\lambda<K)$ be the domain in which $W(z)<\lambda$. Then $D_{\lambda}$ determines a set of ideal boundary of capacity zero. Thus we can easily

3) Z. Kuramochi: On covering surfaces, Osaka Math. Jour. (1953). 
prove that $\int_{\partial D_{\lambda}} \frac{\partial \omega^{\prime}}{\partial n} d s=\int_{\partial R_{0}} \frac{\partial \omega^{\prime}}{\partial n} d s$, whence the length of the image of $\partial D_{\lambda}=2 \cdot 2 \pi \lambda$. Let $G$ be a non compact domain of $\left(D-R_{n_{0}}\right)+\left(D-R_{n_{0}}\right)$ lying over $1<|W|<K$ and $0<\arg W<\pi$. Let $U_{n}(z)$ be a harmonic function in $\left(D-R_{n_{0}}-\left(G \cap D_{\lambda}\right)\right)\left(D-R_{n_{0}}-\left(G \cap D_{\lambda}\right) \hat{)}\right.$ such that $U_{n}(z)=0$ on $\partial R_{n_{0}}+\partial \hat{R_{n_{0}}}, U_{n}(z)=1$ on $\partial D_{\lambda} \cap G$ and has the minimum integral. On the other hand let $U_{n}^{\prime}(z)$ be a harmonic function in the ring $1 \leqq|W|<\lambda$ with radial slits above-mentioned such that $U^{\prime}{ }_{n}(W)=1$ on $|W|=\lambda, 0<\arg W<\pi$ and $U^{\prime}{ }_{n}(W)$ has the minimum Dirichlet integral. Then

Therefore by theorem

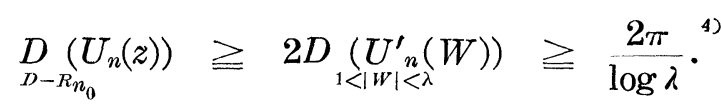

$$
D\left(\lim U_{n}(z)\right)=\operatorname{Cap}\left(B_{D}\right) \geqq \frac{2 \pi}{\log K}>0 . \quad \text { Hence } \lim _{n} \bar{U}_{z \in G}(z)=1 .
$$

On the other hand, let $V_{n}(W)$ be a harmonic function on the ring without radial slits such that $V_{n}(W)=1$ on $|W|=\lambda, 0<\arg W<\pi$ and $V_{n}(W)$ has the minimum Dirichlet integral. Then clearly

$$
D(U(z)) \leqq 2 D\left(\lim _{n} V_{n}(W)\right)<2 D(\omega(z))=\frac{4 \pi}{\log K} .
$$

Therefore on $D+\hat{D}$, there exists a non-constant bounded and Dirichlet bounded harmonic function, because, if it were not so $U(z)$ must be a multiple of $\omega(z)$.

4) Z. Kuramochi: On the behaviour of analytic functions on abstract Riemann surfaces to appear in Ann. Sci. Acad. Fenn. 